\title{
Parallel, independent attentional control settings for colors and shapes
}

\author{
Maha Adamo, Simon Wozny, Jay Pratt, and Susanne Ferber \\ University of Toronto, Toronto, Ontario, Canada
}

\begin{abstract}
Attentional capture can be contingent on attentional control settings (ACSs), such that peripheral cues influence processing for a subsequent target only when they share a critical feature with the target. Our previous demonstration that two ACSs from within the same feature category can be maintained simultaneously allows us to investigate the processing stage at which such ACSs are implemented. We compared the relative efficacy of ACSs from two different feature categories (shape and color) that are associated with different levels within the visual processing hierarchy. Participants were instructed to respond to one of two colors at one location and one of two shapes at another location, while ignoring the nontarget color and shape. We observed that spatial capture was modulated by whether the cues fit an ACS, with slightly greater contingent capture effects for ACSs defined by color than by shape. Thus, two ACSs from different feature sets (color and shape) can be maintained in parallel, although effectiveness of the control set varied with the type of feature.
\end{abstract}

Typically, when a stimulus suddenly appears in the periphery, attention automatically shifts to that location, resulting in preferential processing for events that occur at that location shortly thereafter. Although this stimulus-driven attentional capture, or involuntary orienting, was initially considered to be a bottom-up process that is immune to an individual's objectives (e.g., Yantis \& Jonides, 1984), a continually growing body of literature shows that an individual's current behavioral goals can modulate attentional capture. Beginning with Folk, Remington, and Johnston (1992), there is now considerable evidence that a variety of target-defining features may generate an attentional control set (ACS), which limits engagement of involuntary attentional orienting to stimuli that share a critical feature with the behavioral goal (termed contingent capture; Folk et al., 1992). Feature dimensions that can induce an ACS include visual properties (e.g., color and shape) and temporal properties (e.g., apparent motion and abrupt onset) of stimuli (Folk, Remington, \& Wright, 1994). As long as the target is defined by a particular feature (e.g., green) rather than a class of features (e.g., color), the resulting ACS is feature specific (Folk \& Anderson, 2010; Moore \& Weissman, 2010), and although stimuli may contain multiple features, only those that meet the criteria of the ACS elicit contingent cuing effects (Pratt \& McAuliffe, 2002).

We recently demonstrated that two ACSs can be maintained simultaneously when each is anchored to a distinct region of space (Adamo, Pun, \& Ferber, 2010; Adamo, Pun, Pratt, \& Ferber, 2008). In these studies, participants were asked to respond to blue targets only on the left and to green targets only on the right, generating blue-left and green-right control sets. We presented nonpredictive cues that were congruent or incongruent with respect to the color and/or location of the impending target and observed speeded responses when targets followed fully congruent cues (e.g., a left blue cue preceded a left blue target) and lengthened response times (RTs) when targets followed fully incongruent cues (e.g., a right green cue preceded a left blue target), demonstrating both the benefit and the cost of contingent spatial orienting. Targets following partially congruent cues (e.g., a left green cue or a right blue cue preceded a left blue target) produced RTs that were no different from those for targets that were preceded by a neutral cue or no cue at all, showing that capture occurred only when both color and location of the cue matched the ACS. These contingent capture effects are comparable whether the ACS-related cues appear at either placeholder in isolation (Adamo et al., 2008) or with concurrent neutral cues at the opposite placeholder (Adamo et al., 2010). These results provided evidence both for the flexibility of attentional allocation over multiple goals/ regions and for the specificity of ACSs to maintain one target rule per region.

Our demonstration that distinct ACSs can be maintained simultaneously affords the opportunity to further probe the relative efficacy of features from various categories to instantiate control sets. By characterizing which feature categories can generate an ACS and specifically comparing the relative specificity of ACSs from various feature categories that are associated with different levels of visual processing, we can better understand the level at which contingent capture operates. If an ACS functions

M.Adamo,maha@psych.utoronto.ca 
by facilitating bottom-up priming of features, contingent orienting should be commensurate across feature categories insofar as the different categories elicit comparable levels of perceptual priming. For example, previous work has shown that perceptual priming for colors occurs at much earlier levels of processing within the visual hierarchy than does perceptual priming for form (Breitmeyer, Ogmen, \& Chen, 2004). Thus, if an ACS is effective at the level of perceptual priming, we should find that only color ACSs are effective, with no contingent capture for ACSs defined by shape. Alternatively, if ACSs operate at later stages of visual processing, such as in the activation of target representations within working memory (Moore \& Weissman, 2010; Parrott, Levinthal, \& Franconeri, in press), the relative efficacy of ACSs within different feature classes should be dependent on the specificity of working memory representations, such that the degree of contingent capture reflects how closely a cue fits a target template (Anderson \& Folk, 2010). Although the basic observation of contingent capture may not differ across feature categories, the extent of contingent orienting may differ by category because of differences in working memory capacity for features within different categories (Alvarez \& Cavanagh, 2004).

In previous studies of contingent capture in which two feature classes were pitted against one another (e.g., Folk et al., 1992; Folk et al., 1994; Theeuwes, 1990), the researchers were not able to directly compare capture effects across feature categories because, by necessity, targets within a block were always defined with respect to only one feature category at a time. Thus, any comparisons between target classes had to be conducted indirectly between blocks. Our dual ACS paradigm, however, provides the opportunity to probe responses to targets that are defined with respect to distinct categories simultaneously, which allows for a direct comparison of the magnitude of capture by different feature classes within the same context. In the present study, we presented two feature categories, colors and shapes, with one target and one nontarget feature in each. As in our previous studies (Adamo et al., 2010; Adamo et al., 2008), two ACSs were defined by the distinct target response requirements, and nonpredictive cues containing either the target or the nontarget feature were presented. To compare the specificity of ACSs defined by features of different categories, we modified the design such that there was no overlap between where features of either category could appear, with colors always appearing on one side and shapes on the other. This established two completely separate ACS, thereby allowing us to contrast the specificity of a color ACS directly with that of a shape ACS. Thus, we could examine the capture effects that either feature class exerted independently, with no overlap between features appearing at either location (i.e., the relevance of a given feature was not contingent on the location at which it appeared). In other words, with this design, we could directly compare spatial capture between completely independent ACSs defined by features from two distinct categories maintained in parallel.

\section{METHOD}

\section{Participants}

Twenty-nine participants were recruited from the University of Toronto either for bonus course credit or for monetary remuneration. Of these, 4 were excluded (see the Results section for details), with data from 25 participants ( 21 female) contributing to the final analyses. These participants ranged in age from 18 to 30 years, with a mean age of 22.0 years $(S D=3.0$ years), and had normal or corrected-to-normal vision. All but 2 were right-handed.

\section{Apparatus}

Stimuli were presented on a Viewsonic G220fb flat-screen CRT monitor with $40.5 \mathrm{~cm}$ of viewable screen and a $65-\mathrm{Hz}$ refresh rate. The experiments were programmed and presented using Presentation software (Version 11.0, www.neuro-bs.com). A chinrest was used to ensure a viewing distance of $57 \mathrm{~cm}$. All responses were made with the right hand on the space bar of a standard keyboard positioned at a comfortable distance on the table.

\section{Stimuli and Procedure}

Targets and nontargets consisted of filled blue boxes $\left(1.2^{\circ}\right.$ wide), green boxes $\left(1.2^{\circ}\right.$ wide), gray triangles $\left(1.5^{\circ}\right.$ wide $)$, and gray circles $\left(1.2^{\circ}\right.$ wide). The color targets and nontargets were always presented on one side, and the shape targets and nontargets were presented on the other, with side counterbalanced across participants (i.e., half of the participants saw only blue and green squares in the left placeholder and only gray triangles and circles in the right placeholder, and vice versa for the other half). Cues were outlines of the same shapes magnified five times and positioned around a placeholder. As with the targets/nontargets, color cues were always presented on one side, and shape cues on the other.

Each trial began with a blank black screen for an intertrial interval (ITI) of $500 \mathrm{msec}$, after which the initial display appeared, containing a central fixation cross (subtending $1^{\circ}$ of visual angle) and two shaded placeholders centered at $8^{\circ}$ eccentricity to the left and right (each subtending $2^{\circ}$ ). After $1,000 \mathrm{msec}$, a cue appeared at either placeholder for $100 \mathrm{msec}$, followed by a 100 -msec delay. Next, the target or nontarget was presented in either placeholder for $100 \mathrm{msec}$ (see Figure 1A). The trial ended and the ITI was initiated as soon as a response was made or at $2,000 \mathrm{msec}$ from the start of the trial in no-response trials. There were 480 trials evenly divided among all cue and target/nontarget combinations and an additional 20 practice trials at the start that were not included in the analyses.

The participants were instructed to keep their eyes centrally fixated, to ignore cues when they appeared, and to respond as quickly and as accurately as possible to targets depending on their identity. The participants were informed that shapes would appear only on one side and colors only on the other and were then given one target rule per side: For example, a participant who saw gray shapes on the left and colored boxes on the right was instructed to respond only to triangles on the left and only to blue on the right and to withhold responses both to circles on the left and to green on the right (i.e., left triangle and right blue control sets). Just as we counterbalanced the side to which the colors and shapes were assigned, the specific color and shape to which a participant should respond were also counterbalanced (with targets and nontargets equally likely to appear on either side)

Although gray shapes always appeared on one side and colored boxes on the other, both the location and the identity of the cue were completely nonpredictive. Cues were classified according to whether they fit an ACS $(\mathrm{F}+)$ or not $(\mathrm{F}-)$ and whether they appeared on the same side as $(\mathrm{S}+)$ or on the opposite side from $(\mathrm{S}-)$ the target (see Figures $1 \mathrm{~B}$ and $1 \mathrm{C}$ ). Thus, we tested trials with four cue types: (1) F+S + , where the cue fit an ACS and appeared at the target's location (e.g., a triangle cue appearing on the left for the example provided above with a triangle target); (2) $\mathrm{F}+\mathrm{S}-$, where the cue fit an ACS but did not appear at the target's location (e.g., a 
A

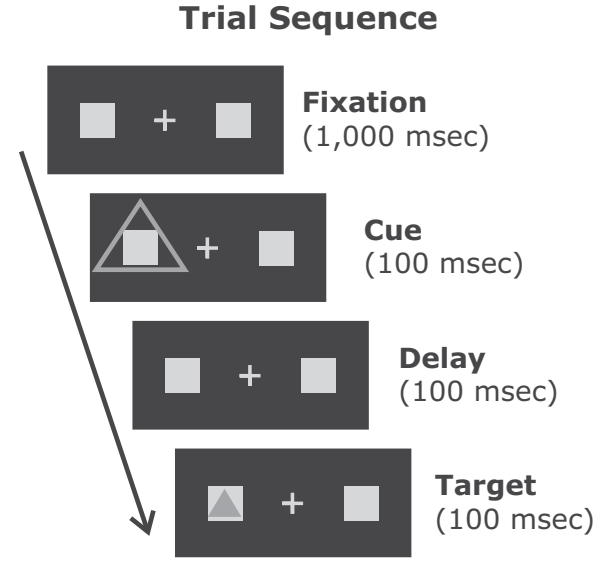

B

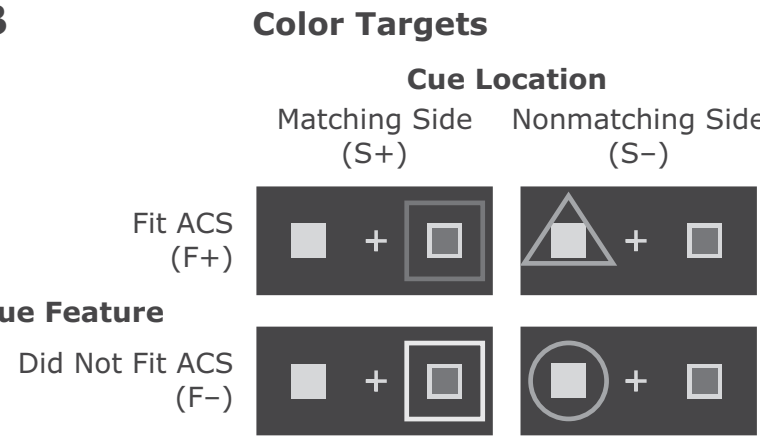

C

Cue Feature

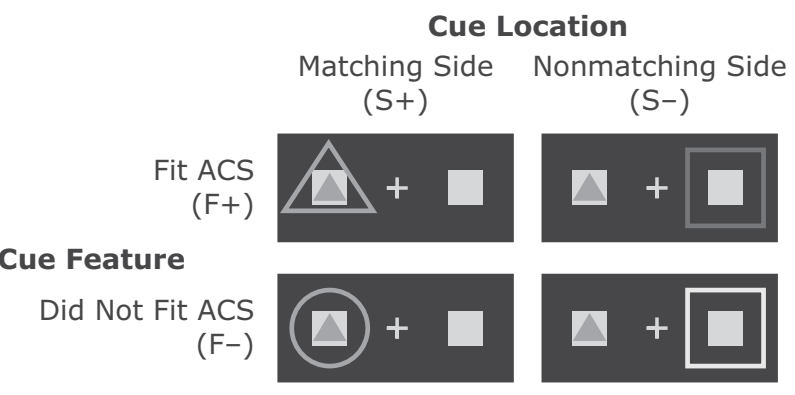

Figure 1. Examples of trials assuming left triangle and right blue attentional control sets (ACSs), in which participants would respond to gray triangles on the left while ignoring gray circles, and to blue squares (represented as the darker gray) on the right while ignoring green squares (represented as white). (A) Illustration of a single trial sequence, in this case an $\mathrm{F}+\mathrm{S}+$ trial with a triangle target. (B) All four cue types for color targets. (C) All four cue types for shape targets. Note that colors and shapes do not overlap, but a target in either category is equally likely to be preceded by a cue from the same category or from the other category.

blue cue on the right before the triangle target); (3) $\mathrm{F}-\mathrm{S}+$, where the cue did not fit an ACS but appeared at the target's location (e.g., a circle cue on the left before the triangle target); and (4) $\mathrm{F}-\mathrm{S}-$, where the cue did not fit any ACS and also did not appear at the target's location (e.g., a green cue on the right prior to the triangle target). Note that according to the contingent capture hypothesis, spatial capture effects should be maximal for $\mathrm{F}+$ trials, where the cue fit an ACS $(\mathrm{F}+\mathrm{S}+$ and $\mathrm{F}+\mathrm{S}-$ should have greater capture than $\mathrm{F}-\mathrm{S}+$ and $\mathrm{F}-\mathrm{S}-$ trials $)$.

\section{RESULTS}

The participants' data were excluded if the hit rate for targets was less than $90 \%$ or if the false alarm rate for nontargets to which no response was required was more than $20 \%$, resulting in the exclusion of 4 participants. For the remaining 25 participants, the hit rate averaged at $98.5 \%$ across all trials, with no significant difference between color and shape targets ( $98.8 \%$ and $98.2 \%$, respectively) $[t(24)=1.24, p>.2]$, and the false alarm rate averaged at $5.5 \%$ across all trials $(5.0 \%$ for color nontargets and $6.0 \%$ for shape nontargets) $[t(24)=0.97, p>.3]$. In addition, anticipatory (RTs $<150 \mathrm{msec}$ ) and extremely delayed (RTs $>1,500 \mathrm{msec}$ ) responses were excluded from analyses, resulting in the removal of an average of $0.56 \%$ of all trials across all participants (maximum of $4.0 \%$ for any single participant, $0.52 \%$ for color targets and $0.61 \%$ for shape targets) $[t(24)=0.54, p>.5]$. Overall, hit rate, false alarm rate, and RTs corresponded to previously reported patterns.

The RTs for correct trials were submitted to a target type (color vs. shape) $\times$ cue location ( + vs. $S-$ ) $\times$ ACS $(\mathrm{F}+$ vs. $\mathrm{F}-$ ) ANOVA (see Figures $2 \mathrm{~A}$ and $2 \mathrm{~B})$. The effect of target type did not reach significance $[F(1,24)=$ $2.41, M S_{\mathrm{e}}=2,463.94, p>.1$ ], showing that RTs did not differ overall for color versus shape targets. Significant main effects were found for cue location $[F(1,24)=$ $\left.52.65, M S_{\mathrm{e}}=2,992.15, p<.0001\right](\mathrm{S}+$ cues had shorter RTs than did $\mathrm{S}-$ cues $)$ and ACS $\left[F(1,24)=5.23, M S_{\mathrm{e}}=\right.$ 766.76, $p<.05](\mathrm{F}+$ cues had shorter RTs than did Fcues). No interaction between cue location and target type was found $\left[F(1,24)=2.71, M S_{\mathrm{e}}=755.04, p>.1\right]$, although the target type $\times$ ACS interaction was significant $\left[F(1,24)=5.89, M S_{\mathrm{e}}=568.72, p<.05\right](\mathrm{F}+$ cues had a proportionately greater effect relative to $\mathrm{F}-$ cues for color targets than for shape targets). Most importantly, a significant interaction between location of the cue and ACS was found $\left[F(1,16)=31.28, M S_{\mathrm{e}}=509.00, p<.0001\right]$, because cues that fit an ACS $(\mathrm{F}+)$ produced larger benefits and costs on subsequent target processing than did cues that did not fit an ACS (F-). Finally, the three-way interaction reached significance $\left[F(1,24)=7.04, M S_{\mathrm{e}}=\right.$ $554.00, p<.05$ ], indicating that the difference between $\mathrm{F}+$ and $\mathrm{F}-$ cues was proportionately greater for colors than for shapes specifically when the cue appeared on the same side as the impending target.

To better examine the magnitude of spatial capture, we computed the difference, for both color and shape targets, between target RTs following opposite-sided (S-) and same-sided $(\mathrm{S}+)$ cues when the cue either fit an ACS $(\mathrm{F}+)$ or did not $(\mathrm{F}-)$ (see Figure 3). Although any spatial cue elicited some degree of capture since all $\mathrm{S}+$ trials led to speeded RTs relative to $\mathrm{S}-$ trials $[F(1,24)=52.65$, $\left.M S_{\mathrm{e}}=5,984.31, p<.0001\right]$, our primary interests were whether cues that fit an ACS conferred greater benefits or costs on subsequent target processing and whether the spatial capture effects differed for shapes versus colors. Thus, we submitted the spatial capture scores to a target type (color vs. shape) $\times$ ACS $(\mathrm{F}+$ vs. F-) ANOVA. Contingent capture was observed as a main effect of ACS 


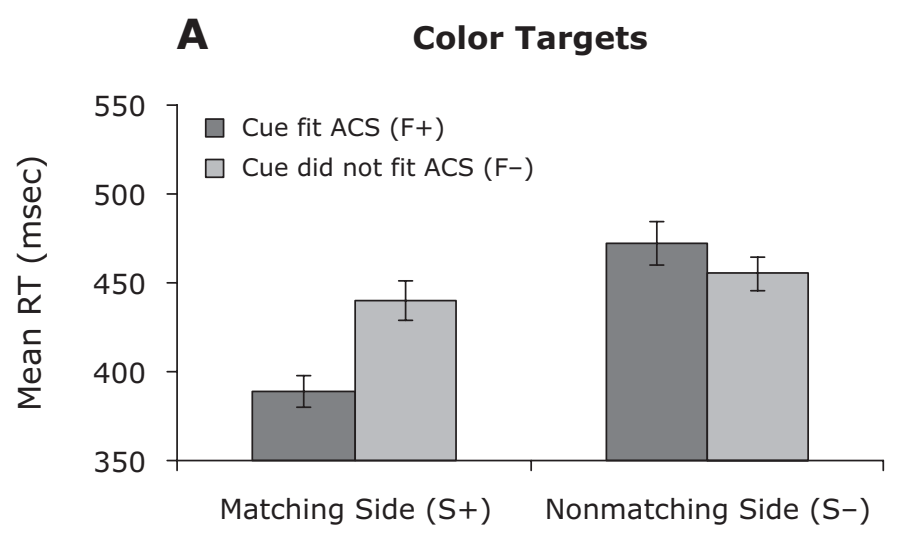

B

Shape Targets

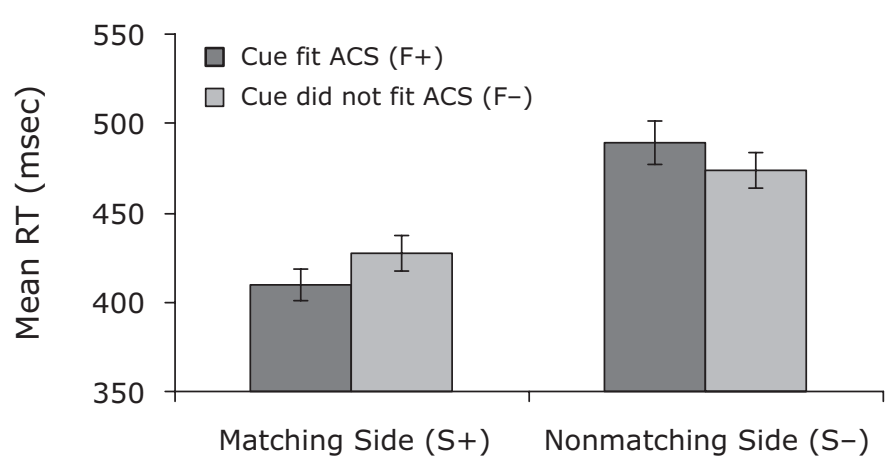

Figure 2. Group mean response times (RTs) for target detection, with error bars representing one standard error of the mean, for $(A)$ color targets and (B) shape targets. The greatest benefits and costs were predicted and observed for targets following cues that fit an attentional control set $(\mathrm{ACS})(\mathrm{F}+)$, represented by the darker bars.

$\left[F(1,24)=53.74, M S_{\mathrm{e}}=1,220.49, p<.0001\right]$. Again, there was no main effect of target type $[F(1,24)=2.71$, $\left.M S_{\mathrm{e}}=1,510.08, p>.1\right]$, but the interaction between target type and whether the cue fit an ACS did reach significance $\left[F(1,24)=7.04, M S_{\mathrm{\circlearrowright}}=1,108.01, p<.05\right]$, because

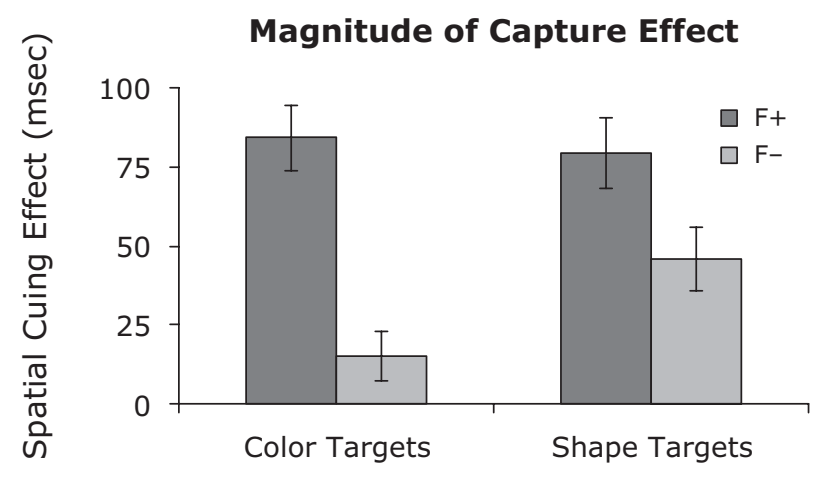

Figure 3. Group mean of the magnitude of spatial capture for color and shape targets, with error bars representing one standard error of the mean, following cues that fit $(F+)$ or did not fit $(F-)$ an attentional control set, represented by the darker and lighter bars, respectively. Although all cues generated some degree of spatial capture, the effect was magnified following $F+$ cues. of a greater degree of spatial capture for $\mathrm{F}$ - cues on samesided shape targets than on color targets.

\section{DISCUSSION}

To summarize, regardless of whether the targets were defined by shape or by color, we found evidence for contingent capture for both ACSs; the relevant cue on each side was more effective in orienting attention as seen in the greater influence on RT following relevant than following irrelevant cues. Both colors and shapes were significantly affected by two co-occurring ACSs. We also found evidence that the magnitude of the effect is demonstrably larger for colors than for shapes. That is, the participants were more likely to orient attention to an irrelevant shape cue than to an irrelevant color cue. This means that sets defined by color may be applied more effectively than those defined by shape, such that the difference in attentional orienting following a relevant versus an irrelevant feature is larger for colors than for shapes (e.g., the participants oriented attention to green but not to blue better than they did to triangles but not to circles).

This study extends our knowledge of the flexibility of attentional control by demonstrating that not only can two 
ACSs be applied when the defining rules specify which features are relevant within a category, but two sets can be maintained when the rules are drawn from distinct feature categories. Early on, Folk et al. (1994) suggested that the specificity of control sets cannot be restricted to feature categories or that colors and shapes may not show dissociable capture effects across categories because both categories fall within the broader class of static discontinuities. Here, we show that this is not the case, because separate ACSs can be generated by features across categories when each target rule depends on one feature from each category. Colors and shapes are both effective at generating ACSs when the specific features are anchored to distinct locations, and cues that fit these ACSs elicit contingent capture that affects target processing at either location.

The present findings provide direct evidence for the parallel maintenance of multiple ACSs due to the independence of the features that constituted targets and nontargets at either location, with colors appearing at one location and shapes at the other. Although our previous studies (e.g., Adamo et al., 2008) did demonstrate capture effects consistent with the application of two ACSs, these sets were not entirely independent, because the relevance of a given feature was contingent on where in the display it appeared. Two mechanisms may have been at play, one corresponding to spatial congruence and the other to feature congruence between cues and targets, but these effects could not be disentangled easily using behavioral measures. For instance, a partially congruent cue may have slowed the response to a target on the opposite side, but in that case, the cue happened to match the target color, which may have actually enhanced target processing; the integration of these two mechanisms would ultimately lead to the observed neutral RT, which is behaviorally indistinguishable from a situation in which that cue simply failed to capture attention at all. This notion is consistent with a recent study in which multiple ACSs were used in a rapid serial visual presentation task and in which the activation of a target representation by a congruent distractor at least partially compensated for the detrimental effect of capture by that distractor (Moore \& Weissman, 2010).

The present task disambiguated spatial and feature congruency: A cue that did not fit the ACS for the location at which it appeared was also completely irrelevant with respect to the ACS on the opposite side. Therefore, we can attribute any difference in cuing effects between relevant and irrelevant stimuli to the attentional control sets applied at each location. We found that spatial cuing effects were not restricted to stimuli that fit an ACS, because even the cues containing nontarget features generated some, albeit a smaller, degree of capture. Importantly, however, the residual effect of cues that fit an ACS was much greater, suggesting that ACSs confer an additional processing benefit after the initial stages of capture. This explanation is supported by our recent electrophysiological study on the maintenance of two ACSs (Adamo et al., 2010), in which we observed that fully congruent cues activated both space- and feature-based attention mechanisms. In that previous work, we made a distinction between these two mechanisms that are independently engaged by separate aspects of the cues. Although only fully congruent cues led to enhanced behavioral performance, we found that even partially congruent cues elicited some later aspects of attentional selection as indexed by the P3 component of the electrophysiological response. Targets following any feature-matching cue (even those that did not meet all contingencies of an ACS) had P3s with increased amplitude. We argued that the P3 effect in that study reflected the activation of a target representation by a cue with the same feature; meanwhile, a separate component, the $\mathrm{N} 2 \mathrm{pc}$, demonstrated the orientation of spatial attention due to any peripheral cue. Again, here, we see that spatial capture was always a factor in this dual ACS task; critically, when the cue contained the target feature that defined the ACS for that feature category, the spatial cuing effects were magnified relative to when the cue contained the nontarget feature and, hence, did not fit an ACS. In other words, space always matters when spatial information is present, but matching an ACS matters even more. On this note, Parrott et al. (in press) recently succeeded in eliminating any spatial contingencies from a task in which two ACSs were maintained, and they confirmed that the feature-based aspect of the cues was effective at a later stage of attentional processing in which target representations were activated, similar to our ERP findings.

The apparent differences in specificity for a colordefined versus a shape-defined ACS or the differences in how effectively each set was applied may be due in part to differences in color being assessed earlier in visual processing than differences in shape, or simply to differences in relative discriminability between the colors versus between the shapes (Theeuwes, 1992). As Anderson and Folk (2010) recently demonstrated, perceptual similarity between cues and targets modulates the magnitude of contingent capture, such that larger cuing effects are observed when the cue and target have a large degree of perceptual overlap or are not highly discriminable. In the present task, the perceptual similarity (or, conversely, the ease of discrimination) between colors and between shapes was not titrated for each participant or selected according to psychophysical protocols. Nonetheless, it seems unlikely that differences in subjective discriminability can account for the asymmetry in color versus shape specificity, given that we found equivalent false alarm rates for color discrimination versus shape discrimination and that the overall RTs for the different target types did not differ. Furthermore, the contingent capture effect for shapes, although smaller than that for colors, was still reliably observed in this study, suggesting that ACSs do not operate on early, bottom-up priming of perceptual representations. A more compelling explanation, particularly given recent work showing the roles of focus within working memory and of target representation priming in contingent capture (Moore \& Weissman, 2010; Parrott et al., in press), is that the instantiation and/or representation of the ACS rules within working memory is better specified for colors than for shapes. That is, our present findings that a color ACS and a shape ACS can be maintained simultaneously, with greater contingent capture effects for the former, indicate 
that attentional control sets operate at a higher level of processing that is subject to the specificity of representations in working memory.

\section{AUTHOR NOTE}

This project was funded by the Natural Sciences and Engineering Research Council, the Canadian Institutes for Health Research, and an Early Researcher Award to S.F. We thank Naseem Al-Aidroos, Katherine Sledge Moore, and our reviewers for their helpful comments. Correspondence concerning this article should be addressed to M. Adamo, 100 St. George Street, Room 4020, Toronto, ON, M5S3G3 Canada (e-mail: maha@psych.utoronto.ca).

\section{REFERENCES}

Adamo, M., Pun, C., \& Ferber, S. (2010). Multiple attentional control settings influence late attentional selection but do not provide an early attentional filter. Cognitive Neuroscience, 1, 102-110. doi:10.1080/17588921003646149

Adamo, M., Pun, C., Pratt, J., \& Ferber, S. (2008). Your divided attention, please! The maintenance of multiple attentional control sets over distinct regions in space. Cognition, 107, 295-303. doi:10.1016/ j.cognition.2007.07.003

Alvarez, G. A., \& Cavanagh, P. (2004). The capacity of visual shortterm memory is set both by visual information load and by number of objects. Psychological Science, 15, 106-111. doi:10.1111/j.0963 -7214.2004.01502006.x

Anderson, B. A., \& FolK, C. L. (2010). Variations in the magnitude of attentional capture: Testing a two-process model. Attention, Perception, \& Psychophysics, 72, 342-352. doi:10.3758/APP.72.2.342

Breitmeyer, B. G., Ogmen, H., \& Chen, J. (2004). Unconscious priming by color and form: Different processes and levels. Consciousness \& Cognition, 13, 138-157. doi:10.1016/j.concog.2003.07.004
Folk, C. L., \& ANDERSON, B. A. (2010). Target uncertainty effects in attentional capture: Color singleton set or multiple attentional control settings? Psychonomic Bulletin \& Review, 17, 421-426.

Folk, C. L., Remington, R. W., \& Johnston, J. C. (1992). Involuntary covert orienting is contingent on attentional control settings. Journal of Experimental Psychology: Human Perception \& Performance, 18, 1030-1044. doi:10.1037/0096-1523.18.4.1030

FolK, C. L., Remington, R. W., \& WRight, J. H. (1994). The structure of attentional control: Contingent attentional capture by apparent motion, abrupt onset, and color. Journal of Experimental Psychology: Human Perception \& Performance, 20, 317-329. doi:10.1037/0096 $-1523.20 .2 .317$

Moore, K. S., \& Weissman, D. H. (2010). Involuntary transfer of a top-down attentional set into the focus of attention: Evidence from a contingent attentional capture paradigm. Attention, Perception, \& Psychophysics, 72, 1495-1509.

Parrott, S. E., Levinthal, B. R., \& Franconeri, S. L. (in press). Complex attentional control settings. Quarterly Journal of Experimental Psychology. doi:10.1080/17470218.2010.520085

Pratt, J., \& McAuliffe, J. (2002). Determining whether attentional control settings are inclusive or exclusive. Perception \& Psychophysics, 64, 1361-1370.

TheEuwes, J. (1990). Perceptual selectivity is task dependent: Evidence from selective search. Acta Psychologica, 74, 81-99. doi:10.1016/0001 -6918(90)90036-F

Theeuwes, J. (1992). Perceptual selectivity for color and form. Perception \& Psychophysics, 51, 599-606.

YANTIS, S., \& Jonides, J. (1984). Abrupt visual onsets and selective attention: Evidence from visual search. Journal of Experimental Psychology: Human Perception \& Performance, 10, 601-621. doi:10 .1037/0096-1523.10.5.601

(Manuscript received February 25, 2010; revision accepted for publication May 22, 2010.) 